\begin{tabular}{c|c|c|c|} 
SELECCIONES MATEMÁTICAS \\
Universidad Nacional de Trujillo \\
ISSN: $2411-1783$ (Online) \\
2021; Vol. 8(2): 348-359.
\end{tabular}

\title{
Results on the Well Posedness of a Distributional Differential Problem
}

\section{Resultados Sobre el Buen Planteamiento de un Problema Diferencial Distribucional}

\author{
Yolanda Santiago Ayala®
}

Received, Oct. 01, 2021

Accepted, Dec. 13, 2021

How to cite this article:

Santiago Y. Results on the Well Posedness of a Distributional Differential Problem. Selecciones Matemáticas. 2021;8(2):348359. http://dx.doi.org/10.17268/sel.mat.2021.02.11

\begin{abstract}
In this work, we study the Fourier Theory in the space of periodic distributions: $P$ '. We analyze the existence of at least one solution for the distributional differential problem in connection with the zeros of a polynomial. We prove that there are infinite solutions when the Fourier coefficients vanish at the integer zeros of the polynomial and otherwise does not have solution. We deduce the existence and uniqueness by considering that the polynomial lacks integer zeros. In the cases of existence, we deduce the analytical solutions. Moreover, we get a result firelated with the continuous dependence of the solution.

Finally, we give some conclusions and applications.
\end{abstract}

Keywords . Distributional differential equation, zeros of a polynomial, existence of solution, periodic distributional space, Fourier inverse transform.

\section{Resumen}

En este trabajo, estudiamos la Teoría de Fourier en el espacio de las distribuciones periódicas: P'. Analizamos la existencia de al menos una solución para el problema diferencial distribucional en conexión con los ceros de un polinomio. Probamos que existen infinitas soluciones si los coeficientes de Fourier se anulan en los ceros enteros del polinomio y si esto no ocurre el problema no posee solución. Si el polinomio carece de ceros enteros se consigue probar la existencia y unicidad de solución. En los casos de existencia de solución, se obtiene la expresión analítica de estas. Además, conseguimos un resultado relacionado con la dependencia continua de la solución.

Finalmente, damos algunas conclusiones y aplicaciones.

Palabras clave. Ecuación diferencial distribucional, ceros de un polinomio, existencia de solución, espacio distribucional periódico, transformada inversa de Fourier.

1. Introducción. Sea la ecuación diferencial, con derivada en el sentido de Fréchet

$$
-i u^{\prime}=g \in C_{p e r}([-\pi, \pi]),
$$

la cual posee infinitas soluciones en $\left.C_{p e r}^{1}(-\pi, \pi]\right)$ si $\widehat{g}(0)=0$, que probaremos en el Teorema 5.2. Nos interesa generalizar este problema en $P^{\prime}$. Así, el problema diferencial distribucional periódico que estudiaremos es

$$
p(D) u=f \in P^{\prime},
$$

donde $p$ es un polinomio de grado $m \geq 1, D: P^{\prime} \longrightarrow P^{\prime}$ es el operador diferencial $D v=-i v^{\prime}$ para $v \in P^{\prime}$ y $f \in P^{\prime}$ es dada. Nos preguntamos si el resultado obtenido para la ecuación diferencial (1.1), sigue siendo válido para (1.2) en $P^{\prime}$. Esta pregunta es propuesta por Iorio en [1], donde se aborda la teoría de

\footnotetext{
*Facultad de Ciencias Matemáticas, Universidad Nacional Mayor de San Marcos, Av. Venezuela S/N Lima 01, Lima, Perú.
} (ysantiagoa@unmsm. edu.pe). 
distribuciones periódicas, ver también [3].

En este artículo analizaremos la existencia de soluciones para (1.2) en función a las raíces del polinomio $p$ y usando la teoría de Fourier en el espacio distribucional periódico. Esto es, probaremos que el problema distribucional (1.2) está bien colocado cuando $p$ no tiene raices enteras. En caso contrario, posee infinitas soluciones cuando los coefientes de Fourier se anulan en las raíces enteras de $p$. Y no posee solución si estos coeficientes de Fourier no se anulan en las raíces enteras de $p$.

El presente artículo está organizado como sigue. En la sección 2, indicamos la metodología usada y citamos el marco funcional, teórico y algunos resultados previos. En la sección 3, introducimos al Operador Diferencial $p(D)$ y probamos importantes resultados. En la sección 4, planteamos el problema y probamos la existencia de solución de (1.2) cuando las raíces del polinomio no son enteras. En la sección 5, probamos la existencia de infinitas soluciones cuando los coeficientes de Fourier se anulan en los ceros del polinomio. Además, probamos que esto generaliza en $P^{\prime}$, de lo que sucede en el caso diferencial de Fréchet. En la sección 6, usando convolución mejoramos la cara de la solución, cuando $p$ carece de ceros enteros. Aquí, resaltemos que la convolución hace de $P^{\prime}$ un álgebra conmutativa con identidad: $2 \pi \delta$. También, probamos la dependencia continua de la solución del problema distribucional cuando $r(p) \cap Z=\emptyset$, donde estamos denotando por $r(p)$ al conjunto de las raíces de $p$. Finalizamos la sección, introduciendo el estudio realizado para problemas diferenciales en $L^{2}([-\pi, \pi])$ y $P^{\prime}-L^{2}([-\pi, \pi])$, respectivamente. Finalmente, en la sección 7 , damos las conclusiones de nuestro estudio.

2. Metodología. Como marco teórico, en este trabajo usamos los siguientes tópicos: Teoría de Fourier en el espacio distribucional periódico, ceros de un polinomio, diferenciación en el sentido de Fréchet y teoría de Operadores. Como referencia en la revisión de algunos resultados previos que usaremos, citamos a Iorio [1], Santiago [2], Santiago and Rojas [4], [5] y [6].

Toda esta teoría la usamos en el estudio de diferenciabilidad distribucional y sus propiedades, análisis de existencia de solución del problema de motivación (1.1), análisis de existencia, unicidad y dependencia continua de solución del problema central (1.2), realizando una serie de cálculos y aproximaciones en el desarrollo del trabajo y mejoras del resultado.

3. El Operador Diferencial $p(D)$. La presentación de los resultados obtenidos lo hemos organizado de la siguiente forma.

3.1. Transformación de un Operador Diferencial en $P^{\prime}$ a un Operador de Multiplicación en $S^{\prime}(Z)$. En esta sección, empezamos mencionando la relación entre transformada de Fourier y derivada distribucional en $P^{\prime}$, y lo que esto genera a nivel de Operadores.

Observación 3.1. Para $f \in P^{\prime}$ tenemos $\left(f^{(n)}\right)^{\wedge}(k)=(i k)^{n} \hat{f}(k), \forall k \in Z, \forall n \in Z^{+}$, entonces al operador diferencial $: f \longrightarrow f^{(n)}$ vía la transformada de Fourier la transforma en un operador de multiplicación: $: \hat{f} \longrightarrow(i k)^{n} \hat{f}$.

3.2. El Operador Diferencial $D$ en $P^{\prime}$. Introducimos el siguiente Operador en el espacio de las distribuciones periódicas.

Definición 3.1. El Operador Diferencial $D$ es definido sobre $P^{\prime}$, es decir, $D: P^{\prime} \longrightarrow P^{\prime}$ y es tal que $f \longrightarrow D f=-i f^{\prime}$. face

Proposición 3.1 (Transformada de Fourier de $D f$ ). El operador Diferencial $D$ es $\mathbb{C}$-lineal y satis-

$$
\widehat{(D f)}(k)=k \hat{f}(k), \quad \forall k \in Z \quad \forall f \in P^{\prime} .
$$

Demostración: En efecto, sea $k \in Z$,

$$
(D f)^{\wedge}(k)=\left(-i f^{\prime}\right)^{\wedge}(k)=-i\left(f^{\prime}\right)^{\wedge}(k)=(-i)(i k) \widehat{f}(k)=k \widehat{f}(k)
$$

Teorema 3.1. El operador Diferencial $D: P^{\prime} \longrightarrow P^{\prime}$ es continuo. Esto es, si $f_{n} \stackrel{P^{\prime}}{\longrightarrow} f$ entonces $D f_{n} \stackrel{P^{\prime}}{\longrightarrow} D f$.

Demostración: Omitiremos la demostración de este teorema dado que es análoga a la prueba del Teorema 3.2, que introduciremos y probaremos posteriormente utilizando la relación (3.2) con $\gamma=1$.

3.3. El Operador Diferencial $p(D)$ en $P^{\prime}$. La composición de operadores, la suma de operadores y el producto por un escalar nos permite introducir el siguiente operador. 
Definición 3.2. Sea $p(z)=\sum_{j=0}^{m} a_{j} z^{j}$ donde $a_{j} \in \mathbb{C}, \forall j=0, \ldots, m$ y $a_{m} \neq 0$, definimos el Operador Diferencial de orden $m$, que denotamos por $p(D)$, como

$$
\begin{aligned}
p(D):=\sum_{J=0}^{m} a_{J} D^{J}: P^{\prime} & \longrightarrow P^{\prime} \\
f & \longrightarrow p(D) f=\sum_{J=0}^{m} a_{J}(-i)^{J} f^{(J)},
\end{aligned}
$$

donde $D^{J}=\underbrace{D \circ \ldots \circ D}_{J-\text { veces }}$ y $D^{0}=I$.

Observación 3.2. Se verifica $p(D) f:=\sum_{J=0}^{m} a_{J} D^{J} f=\sum_{J=0}^{m} a_{J}(-i)^{J} f^{(J)}$.

En efecto, sabemos que $D^{n} f=(-i)^{n} f^{(n)}, \forall n \in N$, luego

$$
\sum_{J=0}^{m} a_{J} D^{J} f=\sum_{J=0}^{m} a_{J}(-i)^{J} f^{(J)}
$$

Proposición 3.2 (Transformada de Fourier de $p(D) f$ ). El operador Diferencial de orden $m: p(D)$ : $P^{\prime} \rightarrow P^{\prime}$ es $\mathbb{C}$-lineal y satisface

$$
(p(D) f)^{\wedge}(k)=p(k) \hat{f}(k), \quad \forall k \in Z, \quad \forall f \in P^{\prime} .
$$

Demostración: Sea $k \in Z$,

$$
\begin{aligned}
(p(D) f)^{\wedge}(k) & =\left[\sum_{J=0}^{m} a_{J}(-i)^{J} f^{(J)}\right]^{\wedge}(k) \\
& =\sum_{J=0}^{m} a_{J}(-i)^{J}\left[f^{(J)}\right]^{\wedge}(k) \\
& =\sum_{J=0}^{m} a_{J}(-i)^{J}(i k)^{J} \widehat{f}(k) \\
& =\sum_{J=0}^{m} a_{J}(k)^{J} \widehat{f}(k)=p(k) \widehat{f}(k)
\end{aligned}
$$

Teorema 3.2. El operador diferencial $p(D): P^{\prime} \longrightarrow P^{\prime}$ es continuo. Esto es, si $f_{n} \stackrel{P^{\prime}}{\longrightarrow} f$ entonces $p(D) f_{n} \stackrel{P^{\prime}}{\longrightarrow} p(D) f$.

Demostración: Si $f_{n} \stackrel{P^{\prime}}{\longrightarrow} f$, por la continuidad de la Transformada de Fourier tenemos que $\widehat{f_{n}} \stackrel{S^{\prime}(Z)}{\longrightarrow}$ $\widehat{f}$, esto es

$$
<\widehat{f_{n}}, \beta>\longrightarrow \widehat{f}, \beta>\text { cuando } n \rightarrow+\infty, \quad \forall \beta \in S(Z)
$$

donde $S(Z)$ es el espacio de las sucesiones rápidamente decrecientes.

Como $<\widehat{f_{n}}, \beta>=\sum_{k=-\infty}^{+\infty} \widehat{f_{n}}(k) \beta_{k}$ y $<\widehat{f}, \beta>=\sum_{k=-\infty}^{+\infty} \widehat{f}(k) \beta_{k}$, tenemos

$$
\sum_{k=-\infty}^{+\infty}\left[\widehat{f_{n}}(k)-\widehat{f}(k)\right] \beta_{k} \longrightarrow 0 \text { cuando } n \rightarrow+\infty, \quad \forall \beta=\left(\beta_{k}\right)_{k \in Z} \in S(Z) .
$$

Por otro lado, tenemos que si $\beta=\left(\beta_{k}\right)_{k \in Z} \in S(Z)$ entonces

$$
\left(k^{\gamma} \beta_{k}\right)_{k \in Z} \in S(Z), \quad \forall \gamma \in Z^{+} .
$$

En efecto, se $m \geq 0$,

$$
\sum_{k=-\infty}^{+\infty}|k|^{m}\left|k^{\gamma} \beta_{k}\right|=\sum_{k=-\infty}^{+\infty}|k|^{m+\gamma}\left|\beta_{k}\right|<\infty, \quad \forall \gamma \in Z^{+}
$$


pues $\beta \in S(Z)$.

Ahora, probaremos que

$$
\left(p(k) \widehat{f_{n}}(k)\right)_{k \in Z} \stackrel{S^{\prime}(Z)}{\longrightarrow}(p(k) \widehat{f}(k))_{k \in Z} \text { cuando } n \rightarrow+\infty .
$$

En efecto, bastará probar que

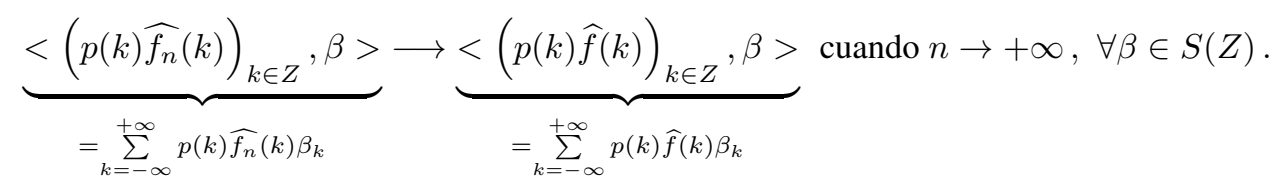

Así, todo se reduce a demostrar que

$$
I_{n}:=\sum_{k=-\infty}^{+\infty} p(k)\left[\widehat{f_{n}}(k)-\widehat{f}(k)\right] \beta_{k} \longrightarrow 0 \text { cuando } n \rightarrow+\infty, \forall \beta=\left(\beta_{k}\right)_{k \in Z} \in S(Z) .
$$

En efecto, sea $\beta \in S(Z)$, usando (3.1) tanto para $\beta$ como para $\left(k^{\gamma} \beta_{k}\right)_{k \in Z} \in S(Z)$, obtenemos

$$
\sum_{k=-\infty}^{+\infty}\left[\widehat{f_{n}}(k)-\widehat{f}(k)\right] k^{\gamma} \beta_{k} \longrightarrow 0 \text { cuando } n \rightarrow+\infty, \forall \gamma \in Z^{+} \cup\{0\}
$$

Observamos que

$$
\begin{aligned}
I_{n}= & a_{0} \sum_{k=-\infty}^{+\infty}\left[\widehat{f_{n}}(k)-\widehat{f}(k)\right] \beta_{k} \\
& +a_{1} \sum_{k=-\infty}^{+\infty}\left[\widehat{f_{n}}(k)-\widehat{f}(k)\right] k \beta_{k}+ \\
& \vdots \\
& +a_{J} \sum_{k=-\infty}^{+\infty}\left[\widehat{f_{n}}(k)-\widehat{f}(k)\right] k^{J} \beta_{k} .
\end{aligned}
$$

Luego tenemos que $I_{n} \rightarrow 0$ cuando $n \rightarrow+\infty$, al ser suma finita de sucesiones convergentes a cero, desde que (3.4) vale para $\gamma=0, \ldots, J$.

Como la inversa de la Transformada de Fourier es continua, obtenemos

$$
p(D) f_{n} \stackrel{P^{\prime}}{\longrightarrow} p(D) f .
$$

4. Planteamiento del Problema Diferencial y Existencia de Solución cuando $r(p) \cap Z=\emptyset$. Planteamos el siguiente problema diferencial en $P^{\prime}$ :

Dado $f \in P^{\prime}, i \exists u \in P^{\prime}$ tal que $u$ es solución de $p(D) u=f$ en $P^{\prime}$ ?, donde la igualdad es entendido en el sentido distribucional.

Esto es, dado $f \in P^{\prime}$ queremos encontrar $u \in P^{\prime}$ tal que

$$
p(D) u=f \quad \text { en } P^{\prime},
$$

(se observa que la ecuación Diferencial (4.1) es de orden $m$ ).

En efecto, supongamos que $u \in P^{\prime}$ es solución de la ecuación (4.1), entonces podemos tomar la transformada de Fourier a la ecuación (4.1) y obtener:

$$
p(k) \hat{u}(k)=\hat{f}(k) \text { para todo } k \in Z .
$$

Si $p$ no tiene raices en $Z$, podemos dividir (4.2) entre $p(k)$ y obtener

$$
\hat{u}(k)=\frac{\hat{f}(k)}{p(k)} .
$$


Luego, si $p$ no tiene raices en $Z$, entonces

$$
u=\left[\left(\frac{\hat{f}(k)}{p(k)}\right)_{k \in Z}\right]^{\vee}=\sum_{k=-\infty}^{+\infty} \hat{u}(k) \phi_{k}=\sum_{k=-\infty}^{+\infty} \frac{\hat{f}(k)}{p(k)} \phi_{k} .
$$

Así, hemos probado: si $u$ es solución de (4.1) entonces la forma explícita de $u$ es dada por (4.3). Así, $u$ expresado por (4.3) es candidato a solución de (4.1) cuando $p$ no tiene raices enteras.

Esto es, si definimos

$$
u:=\left[\left(\frac{\hat{f}(k)}{p(k)}\right)_{k \in Z}\right]^{\vee}
$$

con $f \in P^{\prime}$ y $p$ carente de raices enteras, probaremos que $u \in P^{\prime}$ y que $u$ satisface la ecuación $P(D) u=f$. como

Observamos que $\left(\frac{\hat{f}(k)}{p(k)}\right)_{k \in Z} \in S^{\prime}(Z)$ desde que $\left(\frac{1}{p(k)}\right)_{k \in Z}$ es acotado y $\widehat{f} \in S^{\prime}(Z)$. En efecto,

$$
\lim _{k \rightarrow+\infty} \frac{1}{|a_{o}+a_{1} k+\ldots+\underbrace{a_{m}}_{\neq 0} k^{m}|}=0
$$

entonces $\left(\frac{1}{p(k)}\right)_{k \in Z}$ está acotado.

Sea $k \in Z$, usando esta acotación y que $\widehat{f} \in S^{\prime}(Z)$, tenemos

$$
\left|\frac{\widehat{f}(k)}{p(k)}\right|=\frac{1}{|p(k)|}|\widehat{f}(k)| \leq C_{1} \cdot|\widehat{f}(k)| \leq \underbrace{C_{1} \cdot C}_{\widetilde{C}:=}|k|^{N},
$$

esto es, $\left(\frac{\widehat{f}(k)}{p(k)}\right)_{k \in Z} \in S^{\prime}(Z)$.

Por lo tanto,

$$
\underbrace{\left[\left(\frac{\hat{f}(k)}{p(k)}\right)_{k \in Z}\right]^{\vee}}_{u:=} \in P^{\prime} .
$$

Veremos que $u$ satisface la ecuación.

De la definición de $u$ y como $u \in P^{\prime}$ tenemos

$$
\widehat{u}=\left(\frac{\hat{f}(k)}{p(k)}\right)_{k \in Z} .
$$

Luego, $\widehat{u}(k)=\frac{\widehat{f}(k)}{p(k)}, \forall k \in Z$. Así, $p(k) \widehat{u}(k)=\widehat{f}(k), \forall k \in Z$, esto es

$$
[p(D) u]^{\wedge}(k)=p(k) \widehat{u}(k)=\widehat{f}(k), \quad \forall k \in Z,
$$

desde que $u \in P^{\prime}$. Por lo tanto,

$$
[p(D) u]^{\wedge}=(p(k) \widehat{u}(k))_{k \in Z}=\widehat{f} \in S^{\prime}(Z) .
$$

Tomando la transformada inversa de Fourier tenemos

$$
p(D) u=\left\{[p(D) u]^{\wedge}\right\}^{\vee}=\left\{(p(k) \widehat{u}(k))_{k \in Z}\right\}^{\vee}=\{\widehat{f}\}^{\vee}=f \in P^{\prime} .
$$

Obviamente, de esta prueba se deduce que la solución es única.

Con esto hemos probado los dos siguientes resultados.

Teorema 4.1. Sea $p$ un polinomio sin raices enteras y $f \in P^{\prime}$, entonces el problema Diferencial

$$
p(D) u=f,
$$

posee solución en $P^{\prime}$ y esta solución es única. 
Corolario 4.1. Considerando las hipótesis del Teorema 4.1, la única solución del problema diferencial (4.4) es

$$
u=\left[\left(\frac{\hat{f}(k)}{p(k)}\right)_{k \in Z}\right]^{\vee}=\sum_{k=-\infty}^{+\infty} \frac{\hat{f}(k)}{p(k)} \phi_{k} .
$$

A seguir damos una prueba alternativa referente a la unicidad de solución.

Proposición 4.1 (Unicidad de Solución). Sea $p(k) \neq 0, \forall k \in Z$ y $f \in P^{\prime}$, entonces el problema Diferencial $p(D) u=f \in P^{\prime}$ posee unicidad de solución en $P^{\prime}$.

Demostración: Supongamos que existe $u, v \in P^{\prime}$ solución de $p(D) u=f$ entonces $P^{\prime} \ni w=u-v \mathrm{y}$ satisface $p(D) w=0$. Luego, tomando la transformada de Fourier obtenemos $p(k) \widehat{w}(k)=0, \forall k \in Z$. Si $p(k) \neq 0, \forall k \in Z$, entonces $\widehat{w}(k)=0, \forall k \in Z$. Esto es $\widehat{w}=0$.

Así, $w=\widehat{w}^{\vee}=0$.

\section{Ejemplo 4.1.}

Sea $p(z)=15 z^{2}-2 z-1$, entonces el problema $p(D) u=\delta \in P^{\prime}$ posee una única solución.

Sabemos que $p(z)=6\left(z-\frac{1}{3}\right)\left(z+\frac{1}{5}\right)$. Así, las raices de $p$ son $-\frac{1}{5}$ y $\frac{1}{3}$. Esto es, $p$ no tiene raices enteras.

Aplicando el Teorema 4.1, tenemos que el problema Diferencial posee una única solución en $P^{\prime}$. Aplicando el Corolario 4.1 y usando $\widehat{\delta}(k)=\frac{1}{2 \pi}, \forall k \in Z$, tenemos que la única solución es

$$
u=\left[\left(\frac{1}{2 \pi p(k)}\right)_{k \in Z}\right]^{\vee}=\sum_{k=-\infty}^{+\infty} \frac{1}{2 \pi p(k)} \phi_{k} .
$$

La prueba, en detalle, puede realizarse repitiendo la demostración del Teorema 4.1 para este caso particular.

5. Existencia o no Existencia de Solución del Problema cuando $r(p) \cap Z \neq \emptyset$.

5.1. No existencia de solución de un Problema Diferencial en $P^{\prime}$. Evidenciamos esto con un ejemplo.

Ejemplo 5.1. Sea $p(z)=z . ; \exists u \in P^{\prime}$ tal que $p(D) u=\delta \in P^{\prime}$ ?. Supongamos que exista $u \in P^{\prime}$ tal que $D u=\delta$, i.e. $-i u^{\prime}=\delta$.

Aplicando la tranformada de Fourier, tenemos

$$
k \widehat{u}(k)=(-i)(i k) \widehat{u}(k)=\widehat{\delta}(k), \quad \forall k \in Z .
$$

Para $k \neq 0$ obtenemos $\widehat{u}(k)=\frac{\widehat{\delta}(k)}{k}$ y para $k=0$ tenemos

$$
0=0 . \widehat{u}(0)=\widehat{\delta}(0)=\frac{1}{2 \pi},
$$

lo cual es absurdo.

Luego, no existe $u \in P^{\prime}$ solución de $D u=\delta$.

5.2. Existencia de solución/sin unicidad de solución. Recordemos, estamos denotando por $p(r)$ al conjunto de todas las raices del polinomio $p$.

Teorema 5.1. Si p tiene raiz entera (i.e. $p(r) \cap Z \neq \emptyset$ ) entonces son equivalentes los siguientes enunciados:

1. Existe $u \in P^{\prime}$ solución de

$$
p(D) u=f \quad f \in P^{\prime} .
$$

2. $\hat{f}$ se anula en las raices enteras de $p$ (i.e. $\widehat{f}(k)=0, \forall k \in p(r) \cap Z)$.

Demostración: La prueba es como sigue:

1) $\Rightarrow 2$ )

Si $\exists u \in P^{\prime}$ que sea solución de (5.1), tenemos

$$
p(D) u=f,
$$

entonces tomando la transformada de Fourier, tenemos

$$
\underbrace{[p(D) u]^{\wedge}(k)}_{=p(k) \widehat{u}(k)}=\widehat{f}(k), \quad \forall k \in Z .
$$


Por lo tanto,

$$
p(k) \widehat{u}(k)=\widehat{f}(k), \quad \forall k \in Z .
$$

Si $k_{i} \in p(r) \cap Z$, usando igualdad (5.2) obtenemos

$$
\underbrace{p\left(k_{i}\right)}_{=0} \hat{u}\left(k_{i}\right)=\hat{f}\left(k_{i}\right) .
$$

Luego

$$
\hat{f}\left(k_{i}\right)=0
$$

esto es, $\widehat{f}$ se anula en las raices enteras de $p$.

2) $\Rightarrow 1$ )

Si $\widehat{f}(k)=0, \forall k \in r(p) \cap Z$, probaremos la existencia de solución del problema (5.1).

Supongamos que existe $u \in P^{\prime}$ solución de (5.1), entonces vale (5.2) y usando las hipótesis, observamos:

$$
\underbrace{p(k)}_{=0} \widehat{u}(k)=\underbrace{\widehat{f}(k)}_{=0}, \quad \forall k \in r(p) \cap Z,
$$

entonces

$$
\hat{u}(k) \text { puede tomar cualquier valor en } \mathbb{C}, \quad \forall k \in r(p) \cap Z \text {. }
$$

Además, de (5.2), para todo $k \in Z-r(p)$ vale:

$$
\hat{u}(k)=\frac{\hat{f}(k)}{p(k)} .
$$

Esto es,

$$
u=\sum_{k \in Z-r(p)} \widehat{u}(k) \phi_{k}+\underbrace{\sum_{\text {finito }}^{\sum_{\text {sum }}^{r(p) \cap Z}} \widehat{u}(k) \phi_{k}}_{\text {sumafinita }} .
$$

i.e.

$$
u=\underbrace{\sum_{k \in Z-r(p)} \frac{\widehat{f}(k)}{p(k)} \phi_{k}}_{\in P^{\prime}}+\underbrace{\sum_{k_{i} \in r(p) \cap Z} \underbrace{\widehat{u}\left(k_{i}\right)}_{c_{k_{i}}=} \phi_{k_{i}}}_{c_{k_{1}} \phi_{k_{1}}+\ldots+c_{k_{\gamma}} \phi_{k_{\gamma}} \in P \subset P^{\prime}} .
$$

Así, a $u$ lo denotamos por $u_{c_{k_{1}}, \ldots, c_{k_{\gamma}}}$.

Por lo tanto, $u$ tiene infinitas caras

$$
\left\{u_{c_{k_{1}}, \ldots, c_{k_{J}}}\right\}_{\left(c_{k_{1}}, \ldots, c_{k_{\gamma}}\right) \in \mathbb{C} \gamma} .
$$

Así, (5.9) es candidato a solución de (5.1). En efecto, definimos

$$
u:=\sum_{k \in Z-r(p)} \frac{\widehat{f}(k)}{p(k)} \phi_{k}+\sum_{k \in r(p) \cap Z} \underbrace{\widehat{u}(k)}_{=c_{k}} \phi_{k} .
$$

Obviamente $u \in P^{\prime}$, probaremos que $u$ es solución de (5.1).

En efecto, como $u \in P^{\prime}$, entonces $P^{\prime} \ni p(D) u=\sum_{J=0}^{m} a_{J}(-i)^{J} u^{(J)}$, tomando la Transformada de Fourier tenemos

$$
[p(D) u]^{\wedge}(k)=p(k) \widehat{u}(k), \quad \forall k \in Z
$$


Si $k \in r(p) \cap Z$, usando (5.12) tenemos

$$
\begin{aligned}
{[p(D) u]^{\wedge}(k) } & =p(k) \widehat{u}(k) \\
& =\underbrace{p(k)}_{=0} c_{k} \\
& =0=\widehat{f}(k) .
\end{aligned}
$$

Por otro lado, si $k \in Z-r(p)$, usando (5.12) tenemos

$$
\begin{aligned}
{[p(D) u]^{\wedge}(k) } & =p(k) \underbrace{\widehat{u}(k)}_{=\frac{\hat{f}(k)}{p(k)}} \\
& =\widehat{f}(k) .
\end{aligned}
$$

De (5.13) y (5.14) tenemos

$$
[p(D) u]^{\wedge}(k)=\widehat{f}(k), \quad \forall k \in Z
$$

Por lo tanto,

$$
p(D) u=\left\{[p(D) u]^{\wedge}\right\}=\{\widehat{f}\}^{\vee}=f
$$

Corolario 5.1 ( $\nexists$ unicidad de solución). Si p es un polinomio tal que $r(p) \cap Z \neq \emptyset$ y $\widehat{f}$ se anula en $r(p) \cap Z$, entonces la ecuación diferencial (5.1) posee infinitas soluciones.

Demostración: Se concluyó en (5.10).

También, es importante resaltar que del Teorema 5.1 se deduce el siguiente resultado.

Corolario 5.2 ( A solución). Sea $p$ un polinomio tal que $r(p) \cap Z \neq \emptyset$. Si f no se anula en $r(p) \cap Z$ entonces la ecuación diferencial (5.1) no posee solución en $P^{\prime}$.

Demostración: Consecuencia inmediata del Teorema 5.1.

5.3. Problema Diferencial en el sentido de Fréchet. Desde el punto de vista clásico, estudiaremos el siguiente resultado:

Teorema 5.2 (Existencia de $\infty$ soluciones en el sentido de Fréchet). Sea $g \in C_{\text {per }}([-\pi, \pi])$ y $f$ una solución de la ecuación diferencial (con derivada en el sentido de Fréchet)

$$
D f=-i f^{\prime}=g \text {. }
$$

Entonces son equivalentes los siguientes enunciados:

1. f es periódica.

2. g tiene promedio nulo, i.e. $\underbrace{\frac{1}{2 \pi} \int_{-\pi}^{\pi} g(s) d s}_{=\hat{g}(0)}=0$.

Demostración: Sea $f$ solución de $-i f^{\prime}=g$ con $g \in C_{\text {per }}([-\pi, \pi])$, entonces $f$ es derivable y su derivada es continua y periódica con periodo $2 \pi$. Desde que $g$ es continua, existe la integral de $g$ en el intervalo $\left[x_{o}, x\right]$, luego, podemos integrar la ecuación y obtener

$$
\underbrace{\int_{x_{o}}^{x} f^{\prime}(s) d s}_{=f(x)-f\left(x_{o}\right)}=i \int_{x_{o}}^{x} g(s) d s .
$$

Esto es, la expresión de $f$ es

$$
f(x)=f\left(x_{o}\right)+i \int_{x_{o}}^{x} g(s) d s
$$

Si definimos

$$
f_{c}(x):=c+i \int_{x_{o}}^{x} g(s) d s
$$

donde $c$ es un número fijo y arbitrario. Entonces, derivando se obtiene $f_{c}^{\prime}(x)=i g(x)$, esto es $f_{c}$ satisface la ecuación: $-i f_{c}^{\prime}=g$ y $f_{c}\left(x_{o}\right)=c$. 
Así, obtenemos que

$$
\left\{f_{c}\right\}_{c \in \mathbb{C}} \text { son soluciones del problema diferencial (5.15), }
$$

esto es, el problema en mensión posee infinitas soluciones.

Ahora, probaremos la equivalencia de los enunciados. 1) $\Rightarrow 2$ )

Si $f$ es periódico de periodo $2 \pi$, entonces $f(-\pi)=f(-\pi+2 \pi)=f(\pi)$. Integrando la ecuación tenemos

$$
\begin{aligned}
\frac{1}{2 \pi} \int_{-\pi}^{\pi} g(s) d s & =\frac{1}{2 \pi} \int_{-\pi}^{\pi}(-i) f^{\prime}(s) d s \\
& =\frac{-i}{2 \pi} \int_{-\pi}^{\pi} f^{\prime}(s) d s=\frac{-i}{2 \pi}\left\{\left.f(s)\right|_{-\pi} ^{\pi}\right\} \\
& =\frac{-i}{2 \pi}\{f(\pi)-f(-\pi)\} \\
& =\frac{-i}{2 \pi}\{f(\pi)-f(\pi)\} \\
& =0
\end{aligned}
$$

Segunda prueba.- Usando (5.16) o (5.17) obtenemos

$$
\begin{gathered}
f(\pi)=f\left(x_{o}\right)+i \int_{x_{o}}^{\pi} g(s) d s \\
f(-\pi)=f\left(x_{o}\right)+i \int_{x_{o}}^{-\pi} g(s) d s .
\end{gathered}
$$

Usando $f(\pi)=f(-\pi)$, de las igualdades (5.19) y (5.20) obtenemos

$$
\int_{x_{o}}^{\pi} g(s) d s=\int_{x_{o}}^{-\pi} g(s) d s .
$$

Luego, $\int_{-\pi}^{\pi} g(s) d s=0$.

2) $\Rightarrow 1$ )

Se observa que si $g$ tiene media nula, entonces $\int_{-\pi}^{\pi} g(s) d s=0$, luego usando que $f$ es solución de (5.15), obtenemos

$$
\begin{aligned}
0 & =\int_{-\pi}^{\pi} g(s) d s \\
& =\int_{-\pi}^{\pi}(-i) f^{\prime}(s) d s \\
& =-i \int_{-\pi}^{\pi} f^{\prime}(s) d s=-i\left\{\left.f(s)\right|_{-\pi} ^{\pi}\right\} \\
& =(-i)\{f(\pi)-f(-\pi)\}
\end{aligned}
$$

Esto es, $f(\pi)=f(-\pi)$.

Afirmamos que $f(x+2 \pi)=f(x), \forall x \in \mathbb{R}$.

En efecto, como $g$ es periódica de periodo $2 \pi$ y continua obtenemos

$$
\begin{aligned}
\int_{2 \pi}^{x+2 \pi} g(s) d s & =\int_{0}^{x} g(y+2 \pi) d s \\
& =\int_{0}^{x} g(y) d s .
\end{aligned}
$$

Usando (5.21) obtenemos

$$
\begin{aligned}
\int_{x}^{x+2 \pi} g(s) d s & =\int_{x}^{2 \pi} g(s) d s+\underbrace{\int_{2 \pi}^{x+2 \pi} g(s) d s} \\
& =\int_{x}^{2 \pi} g(s) d s+\int_{0}^{x} g(s) d s \\
& =\int_{0}^{2 \pi} g(s) d s .
\end{aligned}
$$


También,

$$
\begin{aligned}
\int_{0}^{2 \pi} g(s) d s & =\int_{0}^{\pi} g(s) d s+\underbrace{\int_{\pi}^{2 \pi} g(s) d s} \\
& =\int_{0}^{\pi} g(s)+\int_{-\pi}^{0} g(s) d s \\
& =\int_{-\pi}^{\pi} g(s) d s .
\end{aligned}
$$

De (5.22), (5.23) e hipótesis tenemos

$$
\int_{x}^{x+2 \pi} g(s) d s=\int_{0}^{2 \pi} g(s) d s=\int_{-\pi}^{\pi} g(s) d s=0
$$

y desde que

$$
0=\int_{x}^{x+2 \pi} g(s) d s=\int_{x}^{x_{o}} g(s) d s+\int_{x_{o}}^{x+2 \pi} g(s) d s=-\int_{x_{o}}^{x} g(s) d s+\int_{x_{o}}^{x+2 \pi} g(s) d s,
$$

obtenemos

$$
\int_{x_{o}}^{x} g(s) d s=\int_{x_{o}}^{x+2 \pi} g(s) d s .
$$

Sumando $c=f\left(x_{o}\right)$ a la derecha e izquierda en la igualdad (5.24) obtenemos

$$
f(x)=f(x+2 \pi) .
$$

Así, si $g \in C_{p e r}([-\pi, \pi])$ es tal que $g$ tiene media nula, entonces $f \in C_{p e r}^{1}([-\pi, \pi])$.

Corolario 5.3 ( $\exists$ infinitas soluciones). Si $g \in C_{\text {per }}([-\pi, \pi])$ entonces el problema (5.15) posee infinitas soluciones $f_{c} \in C(\mathbb{R})$ tal que $f_{c}^{\prime} \in C_{\text {per }}([-\pi, \pi])$, donde $f_{c}$ es (5.17).

Demostración: Se sigue de (5.18).

Corolario 5.4 ( $\nexists$ unicidad de solución clásica). Si $g \in C_{p e r}([-\pi, \pi])$ tal que $\widehat{g}(0)=\frac{1}{2 \pi} \int_{-\pi}^{\pi} g(s) d s=$ 0 (i.e. $\widehat{g}$ se anula en el cero de $p(z)=z$ ), entonces el problema (5.15) posee infinitas soluciones $f_{c} \in$ $C_{\text {per }}^{1}([-\pi, \pi])$, donde $f_{c}$ es (5.17).

Demostración: Sigue del Corolario 5.3 y desde que $f_{c}$ es periódica, debido al Teorema 5.2.

Observación 5.1. El Corolario 5.4 lo tenemos generalizado en $P^{\prime}$ y lo hemos desarrollado en el Corolario 5.1 .

\section{Aplicación de la Convolución en $P^{\prime}$.}

6.1. Mejoras del Teorema 4.1. A continuación se evidenciará una distribución periódica fundamental en la forma explícita de la solución del problema diferencial.

Proposición 6.1. Sea $f \in P^{\prime}$ y $p$ un polinomio que no tiene raiz entera entonces la solución $u \in P^{\prime}$ del Problema Diferencial: $p(D) u=f$ la podemos expresar como $u=G * f$, donde $G=\left[\left(\frac{1}{p(k)}\right)_{k \in Z}\right]^{\vee} \in P^{\prime}$ y $\widehat{G}=\left(\frac{1}{p(k)}\right)_{k \in Z} \in S^{\prime}(Z)$.

Esto es,

$$
u=\sum_{k=-\infty}^{+\infty} \frac{\hat{f}(k)}{p(k)} \phi_{k}=G * f .
$$

Demostración: En efecto, si $p$ no tiene raiz entera, sabemos que $\left(\frac{1}{p(k)}\right)_{k \in Z}$ está acotada, luego $\left(\frac{1}{p(k)}\right)_{k \in Z} \in$ $S^{\prime}(Z)$. Así, $G:=\left[\left(\frac{1}{p(k)}\right)_{k \in Z}\right]^{\vee} \in P^{\prime}$ tal que $\hat{G}(k)=\frac{1}{p(k)}, \forall k \in Z$.

Como $\widehat{u}(k)=\frac{\widehat{f}(k)}{p(k)}=\widehat{G}(k) \widehat{f}(k)=[G * f]^{\wedge}(k)$ entonces $u=G * f$

Proposición 6.2. Si $r(p) \cap Z=\emptyset$ entonces la ecuación diferencial $p(D) u=2 \pi \delta \in P^{\prime}$ posee como única solución a

$$
G:=\left[\left(\frac{1}{p(k)}\right)_{k \in Z}\right]^{\vee} \in P^{\prime} .
$$

Llamaremos a $G$ "solución fundamental de la ecuación diferencial”.

Demostración: En efecto, $u=G *(2 \pi \delta)=G$ es la única solución. 
6.2. Dependencia continua del Problema Diferencial. Obtenemos la siguiente propiedad.

Proposición 6.3 (Dependencia Continua). Si $r(p) \cap Z=\emptyset$ entonces existe dependencia continua de la solución de $p(D) u=f$ respecto a $f \in P^{\prime}$. Esto es, sean $f_{n}, f \in P^{\prime}$, si $f_{n} \stackrel{P^{\prime}}{\longrightarrow} f$ entonces $u_{n} \stackrel{P^{\prime}}{\longrightarrow} u$, donde $u_{n} \in P^{\prime}$ es la solución de $p(D) u_{n}=f_{n}$ y $u \in P^{\prime}$ es la solución de $p(D) u=f$.

Demostración: Usando la proposición 6.1 tenemos que $u_{n}=G * f_{n}=f_{n} * G$ y $u=G * f=f * G$.

Si $f_{n} \stackrel{P^{\prime}}{\longrightarrow} f$ tenemos

$$
<f_{n}, \varphi>\longrightarrow<f, \varphi>\text { cuando } n \rightarrow+\infty, \quad \forall \varphi \in P .
$$

Por otro lado, sabemos que si $\varphi \in P$ entonces $\underbrace{\widetilde{G}}_{\in P^{\prime}} * \varphi \in P$.

Sea $\psi \in P$, en particular es válida la convergencia (6.1) para $\varphi:=\widetilde{G} * \psi \in P$, esto es

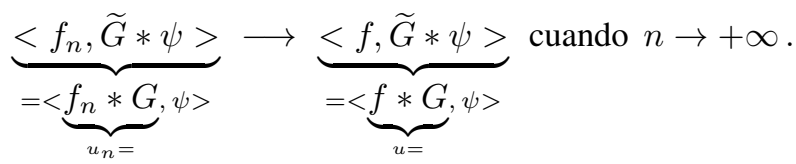

Por lo tanto, $u_{n} \stackrel{P^{\prime}}{\longrightarrow} u$, cuando $n \rightarrow+\infty$.

De los resultados obtenidos podemos evidenciar la siguiente proposición.

Proposición 6.4. Sea p un polinomio y $r(p) \cap Z=\emptyset$ entonces el Operador Diferencial $p(D): P^{\prime} \longrightarrow$ $P^{\prime}$ es lineal, biyectivo, continuo y con inverso continuo.

Demostración: Es consecuencia de los Teoremas 3.2 y 4.1 y de las Proposiciones 4.1 y 6.3.

6.3. Problema Diferencial en $L^{2}([-\pi, \pi])$ y $P^{\prime}-L^{2}([-\pi, \pi])$. Si analizamos el problema diferencial en $L^{2}([-\pi, \pi])$, obtenemos el siguiente resultado.

Teorema 6.1. Sea $z \in \mathbb{C}, p(x)=x^{2}-z$ un polinomio tal que $r(p) \cap Z=\emptyset$ y $f \in L^{2}([-\pi, \pi])$, entonces el problema diferencial

$$
p(D) u=f \text { en } L^{2}([-\pi, \pi])
$$

posee una única solución $u=G_{z} * f \in L^{2}([-\pi, \pi])$ con $u^{\prime \prime} \in L^{2}([-\pi, \pi])$, donde $G_{z}=\left[\left(\frac{1}{k^{2}-z}\right)_{k \in Z}\right]^{\vee} \in$ $C_{p e r}([-\pi, \pi])$, i.e. $G_{z}=\sum_{k=-\infty}^{+\infty} \frac{1}{k^{2}-z} \phi_{k}$, donde la serie converge uniformemente.

Además, considerando $2 \pi \delta \in P^{\prime}-L^{2}([-\pi, \pi])$ en el problema diferencial, obtenemos el siguiente resultado.

Proposición 6.5. Sea $z \in \mathbb{C}, p(x)=x^{2}-z$ un polinomio tal que $r(p) \cap Z=\emptyset$, entonces $G_{z} \in$ $C_{\text {per }}([-\pi, \pi])$ es solución del problema diferencial

$$
p(D) u=2 \pi \delta \text { en } P^{\prime} .
$$

7. Conclusiones. En nuestro estudio del problema diferencial distribucional planteado (4.1) hemos obtenido importantes resultados, entre los cuales destacamos:

1. Usando la teoría de Fourier en $P^{\prime}$, demostramos la existencia y unicidad de solución del problema (4.4) si $p$ no tiene ceros enteros, mostrando así explícitamente a la solución.

2. Probamos la existencia de infinitas soluciones de (5.1) cuando $p$ tiene ceros enteros y $\widehat{f}$ es nulo en dichos ceros.

3. Probamos que si $f$ no se anula en los ceros enteros de $p$, entonces el problema diferencial (5.1) no posee solución.

4. Considerando $p(z)=z$ (i.e. $p$ posee como única raiz entera al cero) y que $\widehat{g}$ se anule en 0 , vemos que el problema diferencial en el sentido de Fréchet (5.15) posee infinitas soluciones. Esta propiedad " $\widehat{f}$ se anule en los ceros enteros del polinomio" fue importante para la existencia de solución, tanto a nivel de derivada de Fréchet como a nivel de derivada distribucional.

5. Usando la convolución en $P^{\prime}$, mejoramos el resultado de existencia de solución (Teorema 4.1 y Corolario 4.1), obteniendo una mejor expresión de la solución en función de la convolución del inverso de Fourier del cociente polinomial con la distribución periódica dato.

6. También, obtenemos la dependencia continua de la solución del problema (4.4). 
7. Finalmente, analizamos la existencia de solución de problemas diferenciales cuando $f \in L^{2}([-\pi, \pi]) \subset$ $P^{\prime}$ y el caso cuando $f=2 \pi \delta \in P^{\prime}-L^{2}([-\pi, \pi])$.

ORCID and License

Yolanda Santiago Ayala https://orcid.org/0000-0003-2516-0871

This work is licensed under the Creative Commons - Attribution 4.0 International (CC BY 4.0)

\section{Referencias}

[1] Iorio R, Iorio V. Fourier Analysis and partial differential equation. Cambridge University; 2001.

[2] Santiago Ayala Y. Tópicos de Análisis Funcional. Fundamentos y Aplicaciones. Alemania, Edit. Acad. Española; 2014.

[3] Santiago Y, Rojas S. Regularity and wellposedness of a problem to one parameter and its behavior at the limit. Bulletin of the Allahabad Mathematical Society. 2017; 32(2):207-230.

[4] Santiago Y, Rojas S. Existencia y Regularidad de solución de la ecuación del calor en espacios de Sobolev Periódico. Selecciones Matemáticas. 2019; 06(01):49-65.

[5] Santiago Y, Rojas S. Existencia y dependencia continua de la solución de la ecuación de onda no homogénea en espacios de Sobolev Periódico. Selecciones Matemáticas. 2020; 07(01):52-73.

[6] Santiago Y, Rojas S. Existence and continuous dependence of the local solution of non homogeneous KdV-K-S equation in periodic Sobolev spaces. Journal of Mathematical Sciences: Advances and Applications. 2021; 64(01):1-19. 